\title{
Evaluation of biventricular volume and systolic function of medium to large ventricular septal defect with pulmonary hypertension in infants aged within 1 year old by real-time three- dimensional echocardiography
}

Jun Gao ( $\sim$ gaojun026@sina.com )

Wuhan Childrens Hospital: Wuhan Women and Children Medical Care Center https://orcid.org/00000001-5746-9334

\section{Xiaoyuan Feng}

Pediatric ultrasound

\section{Li Zhou}

pediatric ultrasound

Jing Kang

pediatric ultrasound

Zhengliang Meng

pediatric ultrasound

Chengwen Guo

pediatric ultrasound

\section{Research}

Keywords: real-time three-dimensional echocardiography, ventricular septal defect, pulmonary hypertension, infant

Posted Date: December 10th, 2020

DOI: https://doi.org/10.21203/rs.3.rs-122923/v1

License: (1) This work is licensed under a Creative Commons Attribution 4.0 International License.

Read Full License 


\section{Abstract}

Background Real-time three-dimensional volume echocardiography could obtain ventricular volume and ejection fraction rapidly and non-invasively without relying on ventricular morphology. Infants under one year of age with medium to large ventricular septal defect $\ \mathrm{VSD} \otimes$ and pulmonary hypertension have the characteristics of recurrent pulmonary infection and feeding difficulties. For these children, early surgery is recommended to reduce pulmonary congestion. Evaluation of biventricular volume and pumping function is important to determine the time of surgery.

Methods In this study 18 children with medium to large VSD complicated with pulmonary hypertension (VSD/PH group ) and 18 healthy children of the same age (control group) were recruited. Biventricular diameter, volume and systolic function were evaluated by two-dimensional echocardiography (2DE), Mmode, tissue Doppler and real-time three-dimensional echocardiography (RT-3DE). The measurements included: left and right ventricular diameter, body surface area standardized ventricular volume (LVEDV/BSA, RVEDV/BSA), LVEF ( M-mode), TAPSE (tricuspid annulus displacement), TAV (tricuspid annulus velocity), LVEF (RT-3DE), RVEF (RT-3DE).

Results Both left and right ventricular diameter and LVEDV/BSA, RVEDV/BSA were significantly larger than those in normal controls $(P<0.05)$. Compared with normal group, LVEF (RT-3DE), RVEF (RT-3DE) and TAV in VSD/PH group decreased $(P<0.05)$. However there were no significant difference in LVEF (Mmode) and TAPSE between the two groups $(P>0.05)$.

Conclusion RT-3DE provides a new approach for noninvasive and rapid assessment of cardiac volume and systolic function for infants with ventricular septal defect and pulmonary hypertension.

\section{Background}

Ventricular septal defect is the most common congenital heart disease with Incidence 1.5-2 per thousand, occupying $40 \%$ of congenital heart defects ${ }^{[1]}$. Severe pulmonary artery congestion, pulmonary interstitial, alveolar edema, decreased lung compliance and increased left ventricular load usually occurred in children with moderate and large ventricular septal defect due to massive left-to-right shunt. Children are prone to recurrent pulmonary infection combined with pulmonary hypertension, heart and respiratory failure, etc al ${ }^{[2]}$. Children should be treated for operation as early as possible. Especially in the case that medical treatment could not control severe pulmonary infection and heart failure, emergency surgery were needed to block left to right shunt and save children's lives ${ }^{[3]}$.

Conventional two-dimensional and M-mode echocardiography have many established indexes to evaluate left and right ventricular function. However the ventricular ejection fraction assessed by twodimensional echocardiography is still based on the hypothesis of cardiac volume morphology. Also there are indexes assessing the motion of the cardiac regional wall to infer the global ventricular function, though it was inaccurate and incomplete. With the development and clinical application of real-time three-dimensional echocardiography (RT-3DE), the volume of the cardiac chamber and the ejection 
fraction without relying on the geometry of the ventricular cavity could be directly measured, it provided a new way for noninvasive and rapid evaluation of heart function. It would be benificial for diagnosing the cardiac function status and determining the time for surgery.

In this study, RT-3DE was used to evaluate ventricular volume and systolic function in infants within 1 years old who with moderate to large ventricular septal defect and pulmonary hypertension.

\section{Methods}

The study was approved by the Local Ethics Committee of our institution. All subjects gave written informed consent.

Study population

18 children with ventricular septal defect and pulmonary hypertension (VSD/PH group) and 18 healthy children of the same age (control group) were recruited in this study. The inclusion criteria as follows : \simple ventricular septal defect or ventricular septal defect with foramen ovale (the width of shunt $\leq$

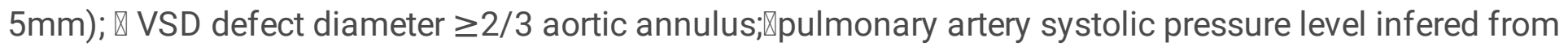
tricuspid regurgitation $\geq 30 \mathrm{mmHg}$ and/or pulmonary artery diastolic pressure level infered from pulmonary regurgitation $\geq 20 \mathrm{mmHg}$; exclusion criteria : $\triangle \mathrm{VSD}$ accompanied with other cardiac structural malformations; $₫ V S D$ with cardiomyopathy; The children in normal control group were confirmed by clinical history, physical examination, electrocardiogram and two-dimensional echocardiography.

\section{Echocardiography}

Echocardiography was performed with the commercially available IE33 system (Philips Medical Systems, Best, the Netherlands) equiped with probe S5-1, S8-3 and real-time three-dimensional probe X3-1, frequency were $1-5 \mathrm{MHz}, 3-8 \mathrm{MHz}$ and $1-3 \mathrm{MHz}$ respectively. Cardiac dimensions, ventricular function were measured according to published guidelines including: tricuspid annulus peak systolic excursion (TAPSE), tricuspid annulus peak velocity (TRV) (Figure 1). RT-3DE were taken on a parasternal fourchamber view by using X3-1 probe, the left or right ventricular cavity were placed in the center of image. It could obtain $60^{\circ} \times 60^{\circ}, 90^{\circ} \times 90^{\circ}, 101^{\circ} \times 104^{\circ}$ full volume $3 \mathrm{D}$ database by adjusting the line density ranging from high, middle to low. Make sure that left or right ventricular cavity in the sampling volume, and then press Full-Volume key to collect the left or right ventricular full volume imaging(3D data base). The sampling principle is obtaining a complete three-dimensional full-volume database of ventricle with a relative high line density, and the image database were stored on CD for off-machine analysis.

\section{RT-3DE echocardiography}

TomTec workstation LV-analysis and RV-Function software were used to analyze the left or right ventricular 3D volume database offline respectively. LV-analysis: three standard sections of left ventricle including left ventricular apical four-charmber view, two-charmber view and three-charmber view were obtained by cutting and rotating the LV 3D data base. The end-systolic and end-diastolic endocardial 
contours of left ventricle were delineated on the above three views (Figure 2). RV-Function: three orthogonal sections of right ventricle including right ventricular four-chamber view, short axis view, coronal view were obtained by cutting and rotating the RV 3D data base. Firstly on the four charmber view determined the central point of mitral annulus, tricuspid annulus and the apical level of the left ventricular cavity. Secondly the end-systolic and the end-diastolic endocardial contours of the above three sections of the right ventricle were delineated. Chordae tendineae, papillary muscles, and regulatory bundles were included in the delineation (Figure 3). Endocardial contours could be manually adjusted when necessary to optimize boundary position and tracking values were automatically calculated by the software. The left or right ventricular volume model were automatically generated by software and the volume-time curve was displayed by two-dimensional coordinate diagram. Left and right ventricular enddiastolic volume (EDV), end-systolic volume (ESV), stroke volume (SV), ejection fraction (EF) values were also given.

\section{Accuracy and Reproducibility}

\section{LV-analysis and RV-Function accuracy}

In this study, another 14 elderly children without heart disease were selected as the subjects for assessing the accuracy of LV-analysis and RV-Function software, 6 male and 8 female, aged 6-14 years. TomTec 4D cardio-view 2.1 software eight-plane method has been used to analyze the volume of left ventricular cavity previously and proved to have a high accuracy ${ }^{[4]}$. So it was used as the gold standard to evaluate the accuracy of TomTec LV-analysis software. Right chamber volume was measured by MCT as the gold standard for assessing the accuracy of the TomTec RV-Function software. MCT was carried out by using Siemens dual-source Flash CT machine, the image post-processing was performed at Philips EBW workstation to map and calculate the volume of the right ventricle.

\section{LV-analysis and RV-Function reproducibility}

Twelve subjects were randomly selected from the study subjects to estimate the variability of the TomTec LV-analysis and RV-Function software and also the three-dimensional ultrasound measurements were performed by the two same experienced observers successively to calculate the inter-observer variability, each blind to the other's measurements. The other observer made a second measurement two weeks later to calculate the intra-observer variability. The reproducibility was estimated by the mean coeffificient of variance $(\mathrm{CV})$ : the absolute value of the difference between the two measurements / the average value of the two measurements $\times 100 \%$.

\section{Statistical analysis}

Statistical analysis was carried out using SPSS software (Version 15.0; SPSS Inc., Chicago, IL, USA). Continuous variables were presented as mean \pm standard deviation and nominal variables as a number and percentage. Comparison between two groups of continuous data were carried out with independent sample T test. Nonparametric test (Mann-Whitney U test) was used to comparison of anomalous 
distribution and classify variables between two groups. Agreement analysis using Bland-Altman curve analysis. A value of $P<0.05$ was considered statistically significant.

\section{Result}

Age and Physical Indexes

There was no significant difference in age and height between the two groups. However body weight and body surface area (BSA) of patient group were smaller than those of control group. Age and physical indexes for the two groups are shown in Table 1.

Table 1

clinical characteristics

\begin{tabular}{|llll|}
\hline & VSD + PH group $(\mathbf{n = 1 8})$ & Control group $(\mathbf{n = 1 8})$ & Pvalue \\
\hline $\begin{array}{l}\text { Age, months } \\
\text { (minimum, median, maximum ) }\end{array}$ & $2,5.50,33$ & $2,6.50,33$ & 0.542 \\
\hline Male sex, $\mathrm{n}(\%)$ & $6(33)$ & $8(44)$ & 0.637 \\
\hline Height, cm (mean \pm SD) & $66.47 \pm 8.29$ & $68.61 \pm 7.86$ & 0.439 \\
\hline Weight, Kg (mean \pm SD) & $6.64 \pm 1.96$ & $8.39 \pm 1.90$ & 0.011 \\
\hline $\begin{array}{l}\text { Body surface area, } \mathrm{m}^{2} \\
\text { (mean } \pm \text { SD) }\end{array}$ & $0.33 \pm 0.07$ & $0.38 \pm 0.07$ & 0.048 \\
\hline Heart rate, bpm & & & 0.970 \\
\hline
\end{tabular}

Echocardiographic characteristics

The echocardiographic parameters were summarized in Table 2. The ventricular septal defects in VSD + $\mathrm{PH}$ group were medium to large. The proportion of children with bidirectional ventricular shunt was $83.33 \%$ in the study. Almost all the pulmonary systolic and diastolic pressure estimated by tricuspid regurgitation and pulmonary regurgitation were severe pulmonary hypertension. Compared with normal control group, the volume of left and right ventricle in VSD + PH group were significantly higher, while LVEF (RT-3DE), RVEF (RT-3DE), TAV were lower. However LVEF (M-mode) and TAPSE showed no difference in two groups . 
Table 2

Echocardiographic characteristics

VSD + PH group $(n=18) \quad$ Control group $(n=18) \quad P$ value

VSD diameter, $\mathrm{mm} \quad 10.35 \pm 2.55$

$($ mean $\pm S D)$

$\mathrm{PA} / \mathrm{AO}$ ratio

$1.44 \pm 0.25$

$0.90 \pm 0.09$

0.000

Bi-directional shunt, $\mathrm{n}(\%) \quad$ 15(83.33)

$\mathrm{TR}, \mathrm{mmHg}($ mean $\pm \mathrm{SD})$

$75.89 \pm 21.16$

$21.19 \pm 3.56$

0.000

$\mathrm{PR}, \mathrm{mmHg}($ mean $\pm \mathrm{SD})$

$49.17 \pm 21.48$

$10.33 \pm 2.64$

0.000

$\mathrm{LVD}, \mathrm{mm}($ mean $\pm \mathrm{SD})$

$25.90 \pm 5.46$

$22.82 \pm 2.33$

0.044

$\mathrm{RVD}, \mathrm{mm}$ (mean $\pm \mathrm{SD})$

$17.34 \pm 3.11$

$15.13 \pm 2.11$

0.049

TAPSE, cm (mean \pm SD)

$1.75 \pm 0.35$

$1.86 \pm 0.13$

0.341

$\mathrm{TAV}, \mathrm{cm} / \mathrm{s}$ (mean $\pm \mathrm{SD})$

$12.08 \pm 1.88$

$15.28 \pm 1.72$

0.000

LVEF(M-mode),\%

$67.78 \pm 8.87$

$70.18 \pm 6.82$

0.457

$($ mean \pm SD)

LVEDV/BSA, $\mathrm{ml} / \mathrm{m}^{2}$

$48.67 \pm 21.46$

$25.59 \pm 6.96$

0.000

$($ mean \pm SD $)$

RVEDV/BSA, $\mathrm{ml} / \mathrm{m}^{2}$

$55.98 \pm 15.35$

$27.69 \pm 4.37$

0.000

(mean \pm SD)

LVEF(RT-3DE), \%

$50.93 \pm 7.50$

$59.38 \pm 7.24$

0.002

(mean \pm SD)

RVEF(RT-3DE), \%

$45.84 \pm 7.71$

$51.05 \pm 6.90$

0.040

(mean $\pm S D)$

$\mathrm{PA} / \mathrm{AO}$ ratio: ratio of pulmonary trunk diameter to accending Aorta diameter; TR/PR:

tricuspid/pulmonary regurgitation; LVD: left ventricle antero-posterior diameter; RVD: right ventricle transverse diameter; TAPSE: tricuspid annulus peak systolic excursion; TAV: tricuspid annulus peak velocity; LVEDV/BSA and RVEDV/BSA: left/right ventricular end-diastolic volume corrected by BSA; LVEF(M-mode): left ventricular ejection fraction obtained by M-mode; LVEF/RVEF(RT-3DE): left/right ventricular ejection fraction obtained by RT-3DE;

LV-analysis and RV-Function accuracy

LV-analysis : The BA curve analysis showed that TomTec LV-analysis and eight-plane method have good relevance and consistency for left ventricular volume and LVEF, both this two method obtained volumetric 
index and cardiac function by delineating endocardium. Left ventricular end-diastolic volume and endsystolic volume measured by LV-analysis are slightly underestimated compared with the eight-plane method, and the dispersion of left ventricular ejection fraction consistency curve increased (Table 3 , Fig. 4).

Table 3

BA curve analysis for TomTec LV-analysis and 4D cardio-view 2.1 eight plane method

\begin{tabular}{|c|c|c|c|c|}
\hline & & LVEDV(ml) & LVESV(ml) & LVEF(\%) \\
\hline $\begin{array}{l}\text { Comparsion with } 4 \mathrm{D} \text { cardio-view } 2.1 \text { eight } \\
\text { plane method }\end{array}$ & $r$ & 0.99 & 0.96 & 0.81 \\
\hline \multirow[t]{3}{*}{$(n=14 \square)$} & $\mathrm{P}$ & $\nabla 0.01$ & $\nabla 0.01$ & $\otimes 0.01$ \\
\hline & $\begin{array}{l}\text { Mean diff. } \\
\pm S D\end{array}$ & $-3.22 \pm 2.4$ & $\begin{array}{l}-0.11 \pm \\
2.71\end{array}$ & $-1.95 \pm 5.71$ \\
\hline & $95 \% \mathrm{CL}$ & $-7.9 \rrbracket 1.5$ & $-5.4 \rrbracket 5.2$ & $-13.14 \otimes 9.24$ \\
\hline
\end{tabular}

RV-Function: The BA curve analysis showed that RT-3DE volume and ejection fraction of the right ventricle analyzed by RV-function were underestimated compared by MCT, but the correlation was good (Table 4, Fig. 5, 6).

Table 4

BA curve analysis for TomTec RV-Function and MCT

\begin{tabular}{|c|c|c|c|c|}
\hline & & RVEDV(ml) & RVESV(ml) & RVEF(\%) \\
\hline Comparison with MCT & $r$ & 0.90 & 0.91 & 0.78 \\
\hline \multirow[t]{3}{*}{$(n=14)$} & $P$ & $₫ 0.001$ & $\llbracket 0.001$ & $\nabla 0.05$ \\
\hline & Mean diff. $\pm S D$ & $-6.18 \% \pm 11.89 \%$ & $-0.01 \% \pm 13.88 \%$ & $-3.39 \% \pm 9.75 \%$ \\
\hline & $95 \% \mathrm{CL}$ & $-29.49 \% \otimes 17.13 \%$ & $-27.23 \% \bowtie 27.20 \%$ & $-22.49 \% \otimes 15.72 \%$ \\
\hline
\end{tabular}

Intra- and inter-observer variability

Intra- observer variability were LVEDV $6 \pm 5 \%$,RVEDV $6 \pm 3 \%$, LVEF $8 \pm 4 \%$ RVEF $9 \pm 5 \%$, inter-observer variability were LVEDV $11 \pm 10 \%$ RVEDV $10 \pm 5 \%$, LVEF $9 \pm 6 \%$ RVEF $9 \pm 6 \%$, respectively,

indicating that both intra-observer and inter-observer had good repeatability.

Clinical outcome 
Despite clinical pulmonary congestion and cardiac dysfunction, all subjects underwent surgery after 3-5 days anti-inflammatory, cardiac, diuretic therapy when ultrasound diagnosis was done and all recovered well.

\section{Discussion}

For medium to large size ventricular septal defects, due to the presence of moderate to large left to right shunt in infancy, severe pulmonary hypertension usually occured in the early stage, and it is tended to develop recurrent pneumonia, heart failure and Influence children's growth and development, more seriously even life-threatening ${ }^{[5,6]}$. For these children pulmonary infection and heart failure that are difficult to control, the longer the course of disease, the more severe the inflammatory injury of lung tissue,and the more difficult the recovery after surgery. Some research reported that $9 \%$ of children with large ventricular septal defects who do not undergo surgery would die within the age of 1 year ${ }^{[7,8]}$.

Children with VSD and severe pulmonary hypertension usually present bidirectional shunt. By pathological autopsy of infants dying from unrestricted ventricular septal defect at 3 to 6 months, HISLOP et al. founded If it was bi-directional shunt and pulmonary vascular resistance was more than 8 $\mathrm{U} / \mathrm{m}^{2}$, the hypertrophy of medial wall of pulmonary artery was obvious ${ }^{[9]}$. Severe pulmonary vascular disease and pulmonary hypertension in congenital heart disease occured mostly in 2 years old children ${ }^{[10]}$. The pulmonary hypertension and bi-directional shunt in small infancy are mostly caused by pulmonary artery constriction and pulmonary vascular spasmodic state, which could be reversed later. Rabino-Vitch et al. reported 215 cases of congenital heart disease with pulmonary hypertension, the mean pulmonary artery pressure and/or pulmonary vascular resistance decreased to normal at 9 months after operation, but it wasn't related to the severity of pulmonary vascular lesion during operation ${ }^{[11]}$. Early operation could block shunt and reduce pulmonary blood flow, which were effective means to prevent the transion from dynamic pulmonary hypertension to resistance pulmonary hypertension. At the same time, surgery is also helpful to prevent more serious infection and promote recovery. Even if some children have poor anti-inflammatory and heart failure treatment effect, they should actively operated. Therefore nowadays many literature reports that early surgery were beneficial for these children and the results was also encouraged ${ }^{[12,13,14]}$. Our experience in this study also supported this opinion.

Low cardiac output syndrome (LCOS) is one of the serious complications of cardiac surgery. Due to immature of structure, function and metabolism of myocardium, infants usually had poor tolerance to surgical trauma and ischemia. Preoperative assessment of cardiac function and selecting appropriate time for surgery would be beneficial for the reduction of occurcence of post-operative low cardiac output syndrome and arrhythmia. Ventricular volume and ejection fraction reflect the fitness of systemic circulation, pulmonary circulation and cardiac function. Ventricular end-systolic volume, end-diastolic volume, cardiac output and ejection fraction were associated with severity of disease and prognosis ${ }^{[15,16}$, 17]. Large ventricular septal defect was ofen accompanied with chronic cardiac insufficiency before operation. Therefore, comprehensive and timely treatment should be given, which could relieve the 
clinical symptoms and reduce the incidence of pulmonary complications, shorten the time in intensive care unit and hospital, and lower the mortality. Previous studies had shown that right ventricular endsystolic volume and end-diastolic volume tended to increase significantly in patients with pulmonary hypertension, while right ventricular ejection fraction, left ventricular end-diastolic volume and left ventricular output may decrease significantly ${ }^{[18,19]}$. Due to the lack of effective methodology for evaluating right ventricular volume by traditional echocardiography, the evaluation of right ventricular function was confined to the measurement of the atrioventricular valve ring displacment or regional myocardial motion, as an alternative solution for the assessment of right ventricular global function. Also the derivation of left ventricular volume and ejection fraction were depended on the assumption of left ventricular cavity geometry. Real-time 3D echocardiography didn't depend on the assumption of geometric configuration, the RT-3D volume database of ventricular cavity was collected by RT-3DE ultrasonic probe and the ventricular volume and ejection fraction were analyzed by the corresponding software, which had the advantages of rapid, non-invasive, simple and repeatable. It provided a new approach and important basis for clinical evaluation of ventricular volume and function.

The feasibility was confirmed that bilateral ventricular volume and ejection fraction in infants aged within 1 years who with ventricular septal defect (VSD) accompanied by pulmonary hypertension could be evaluated by RT-3DE. The results showed that the left and right ventricular volume increased and the ejection fraction decreased, indicating that the bilateral ventricles were overloaded and the ventricular pumping function decreased. It is suggested that clinical measures should be taken to control blood volume and enhance cardiac function. The LVEF by M-mode acquisition is the most commonly used method to obtain LVEF by traditional clinical ultrasound. The index was obtained by measuring the enddiastolic and end-systolic internal diameters of the left ventricle, and then caculated by the corresponding geometric volume derivation formula. In this study, there was no significant difference between the VSD + $\mathrm{PH}$ children and the normal group, so this index was inaccurate, although the LVEF by M-mode could be gotten quickly. Due to the complexity of the right chamber geometry, it is difficult to assume a single geometry, so the ultrasonic evaluation of the right chamber volume and ejection fraction is lacking. The evaluation of right ventricular function by $2 \mathrm{D}$ echocardiography depended on the systolic displacement distance of tricuspid ring (TAPSE), the peak systolic velocity of tricuspid ring (TAV), and the rate of change of right ventricular area (FAC), among them TAPSE was thought to have the best correlation with right ventricular ejection fraction, but all these above indexes were incomplete and had limitations. 3D volumetric ultrasound makes it possible to assess right ventricular volume and ejection fraction directly. Previous studies have showned RT-3DE has good correlation and consistency with angiography, MRI and radionuclide ventriculography in volume and EF assessment ${ }^{[20,21]}$. Our study showed that the RVEF(RT3DE), TAV decreased, indicating RV pump function decreased, but there was no significant difference for TAPSE between the two groups. Therefore, it was inaccurate to evaluate the global ventricular pump function by assessing the displacement of valve ring, maybe it is due to the change of the shape of right ventricle. .

\section{Study Limitations}


The number of cases in this study was still small, and there was no stratified study on patients with ventricular septal defect with different degrees of pulmonary hypertension. In addition the real-time 3D volume database may be misaligned between images due to the limited frame frequency and the fast heart rate of infants, which could lead to the failure of analysis data. Confined by RT-3DE image resolution, indistinct endocardial boundaries could also lead to overestimation or underestimation of true values when delineating endocardium.

\section{Conclusion}

Bilateral ventricular volume increased and systolic function decreased in infants with mediate and large ventricular septal defect complicated with pulmonary hypertension. Real-time three-dimensional echocardiography provides a new method for left and right ventricular volume and ejection fraction.

\section{Declarations}

\section{Conflict of interest:}

none declared.

\section{Acknowledgement}

Thanks to Professor MingXing Xie of Tongji Medical College of Huazhong University of Science and Technology for his guidance and suggestions in this study.

\section{References}

[1] J.I.E. Hoffman. Incidence of congenital heart disease: I. Postnatal incidence. Pediatr Cardiol, 1995, 16 (3): 103-113.

[2] Mmeh JP, Slonim AD, PoHack MM, et al. Long-stay patients in the pediatric intensive care unit. Crit Care Med, 2001,29 (3): 652-657囚

[3] Zhu Weihua, Li Jianhua, Zhu Xiongkai, et al. Follow-up of long-term postoperative outcomes of large ventricular septal defect combined with pulmonary hypertension. Chin J Pediatr, 2001, 39(10): 628-629.

[4] Fang L, Qian W, Gui HY, et al. Impact of the number of image planes of real-time three-dimensional echocardiography on the accuracy of left atrial and ventricular volume measurments. Ultrasound in medicine and biology, 2008, 34(1): 40-46.

[5] Castaneda AR, Mayer JE, Jonas RA, et al. The neonate with critical congenital heart deasease: repair and surgical challenge. J Thorae Cardiovase Surg, 1989, 98: 869-875区 
[6] Bhatt M, Roth sJ, Kumar RK, et al. Management of infants with large, unrepaired ventricular septal defects and respiratory infection requiring mechanical ventilation. J Thorac Cardiovasc Surg, 2004, 127(5): 1466-1473.

[7] Lam CF, Peterson TE, Croatt AJ, et al. Functional adaptation and remodeling of pulmonary artery in flow-induced pulmonary hypertension. Am J Physiol Heart Cir Physiol, 2005, 289(25): 12334-2341区

[8] Keith J D, Rose V, Collins G, et al. Ventricular septal defect: incidence, morbidity and mortality in various age groups $₫ \mathrm{Br}$ Heart, 1971, 33(Suppl): 81-84『

[9] Hislop A, Haworht SG, Shineboume EA, et al囚Quantitative structural analysis of pulmonary vessels in isolated ventrieular septal defect in infancy $\mathbb{B r}$ Heart, 1975, 8 (1): 9-12】

[10] Zhu XiongKai, Ke Xueqin, Yu Jian, et al. Relationship between postoperative pulmonary artery pressure and operative age in patient with severe pulmonary hypertension of congenital heart disease. Chin J Thorac Cardiovasc Surg, 2003,19(4): 236-237.

[11] Rabinovitch M, Keane JF, Norwood WI, et al. Vascular structure in lung tissue obtained at biopsy correlated with pulmonary hemodynamic findings after repair of congenital heart defects. Circulation, 1984, 69(6): 655-667.

[12] Su zhaokang, Zhu zhongqun, Xu zhiwei, et al. Emergency operation on critical congenital heart defects in children: a report of 250 cases. Chin J Pediatr Surg, 2002, 23(5): 401-403.

[13] Bokaap G, Weerheim J, Kappetein AP, et al『Follow up after surgencal closure of congenital septal defect. Eur J Cardiothorac Surg, 2003, 24: 511-515》

[14] Demirag MK, Keceligil HT, Kolbakir F. Primary surgerical repair of VSD. Asian Cradiovasc Thorac Ann, 2003, 11: 213-216.

[15] Humbert M. The burden of pulmonary hypertension. Eur Respir J, 2007, 30(1): 1-2】

[16] Chin KM, Kim NH, Rubin LJ. The right ventricle in pulmonary hypertension. Coron Artery Dis, 2005,16(1): 13-18.

[17] van Wolferen SA, van de Veerdonk MC, Mauritz GJ, et al. Clinically significant change in stroke volume in pulmonary hypertension. Chest, 2011, 139 (5): 1003-1009.

[18] Hoeper MM, Barberd JA, Channick RN, et alखDiagnosis, assessment and treatment of non-pulmonary arterial hypertension pulmonary hypertension. J Am Coll Cardiol, 2009, 54 (1 Suppl): s85-s96》

[19] Galie N, Seeger W, Naeije R, et al. Comparative analysis of clinical trials and edidence-based treatment algorithm in pulmonary arterial hypertension. J Am Coll Cardiol, 2004, 43(12 Suppl): s85-s88. 
[20] Niemann PS, Pinho L, Balbach T, et al. Anatomically oriented right ventricular volume measurements with dynamic three-dimensional echocardiography validated by 3-Tesla magnetic resonance imaging. J Am Coll Cardiol, 2007, 50(17): 1668-1676.

[21] Johnson TR, Hoch M, Huber A, et al. Quantitation of right ventricular function in congenital heart disease: correlation of 3D echocardiography and MRI as complementary methods. Rofo, 2006, 178(10): 1014-1021.

\section{Figures}

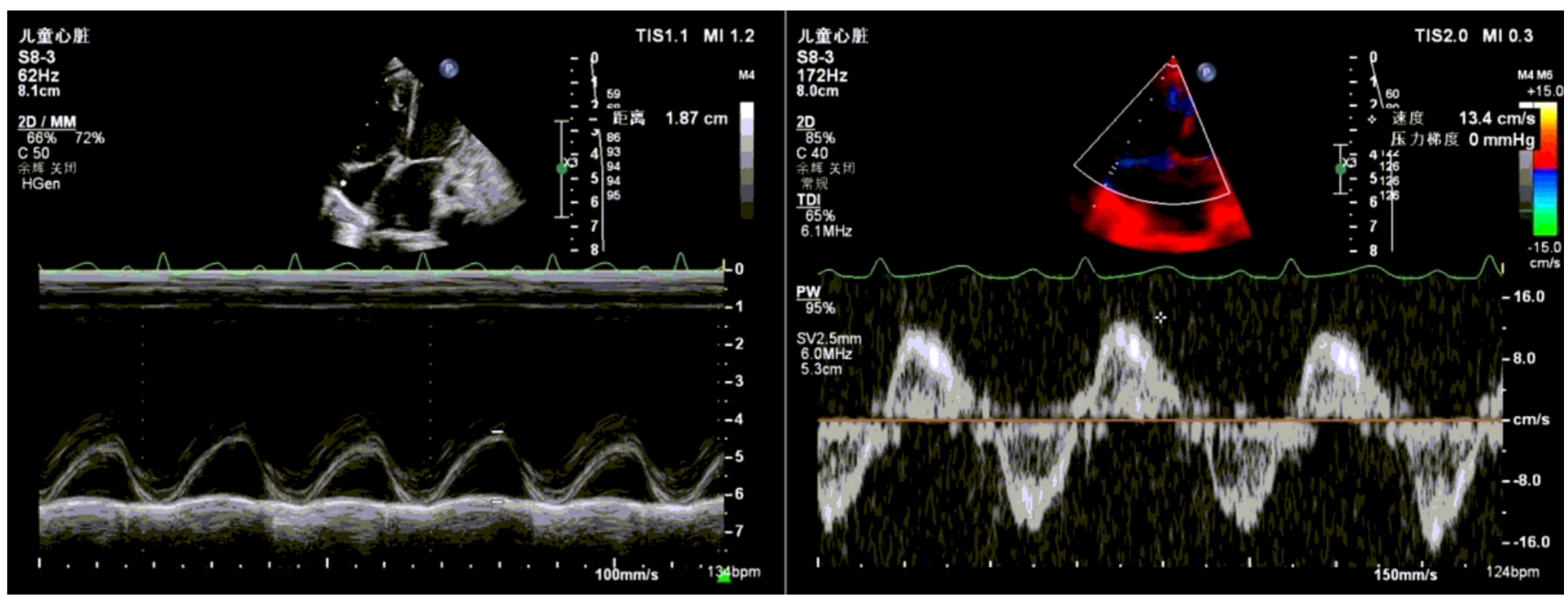

Figure 1

The measurement of tricuspid annulus peak systolic excursion,TAPSE (left) and tricuspid annulus peak velocity ,TAV (right).

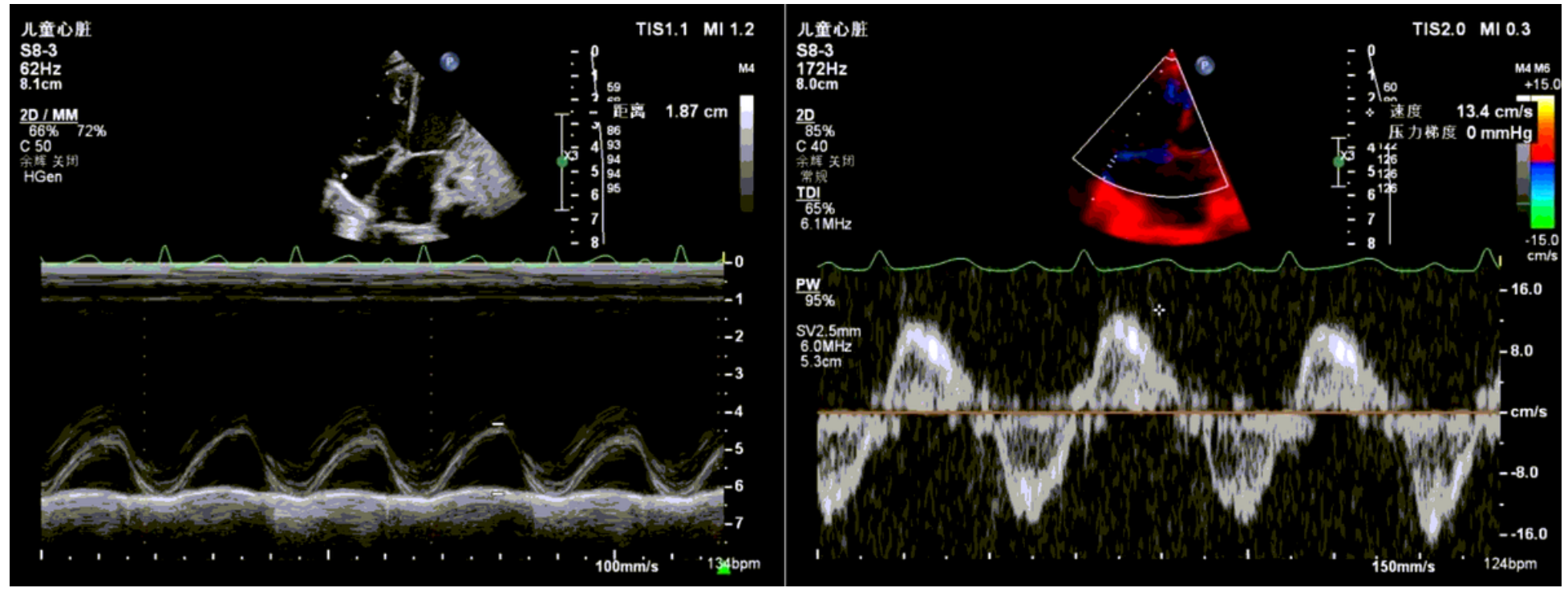

Figure 1 
The measurement of tricuspid annulus peak systolic excursion,TAPSE (left) and tricuspid annulus peak velocity ,TAV (right).

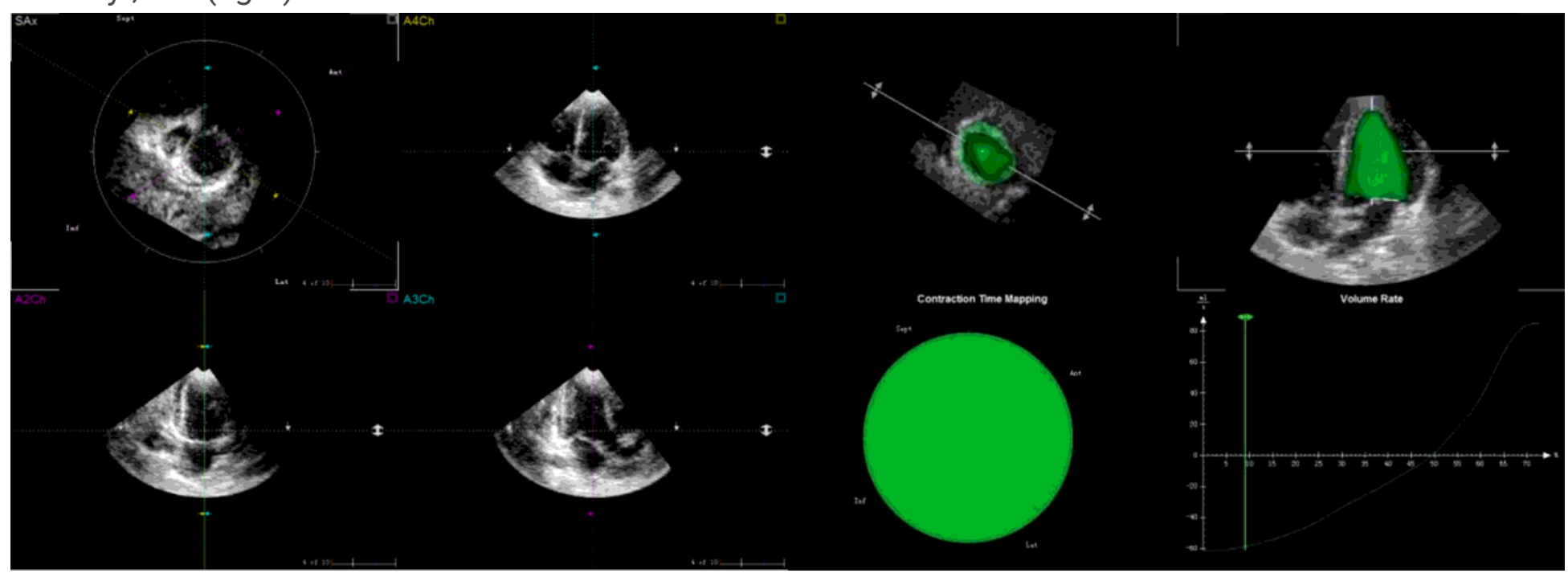

\section{Figure 2}

LV RT-3DE database analysis by TomTec LV-Function. Three standard sections of left ventricle\apical four-charmber view, two-charmber view and three-charmber view were obtained by cutting and rotating the LV three-dimensional data base (left). Left ventricular volume model was automatically generated by delineating endocardium of the three orthogonal sections, and the volume-time curve was also displayed by two-dimensional coordinate diagram (right).

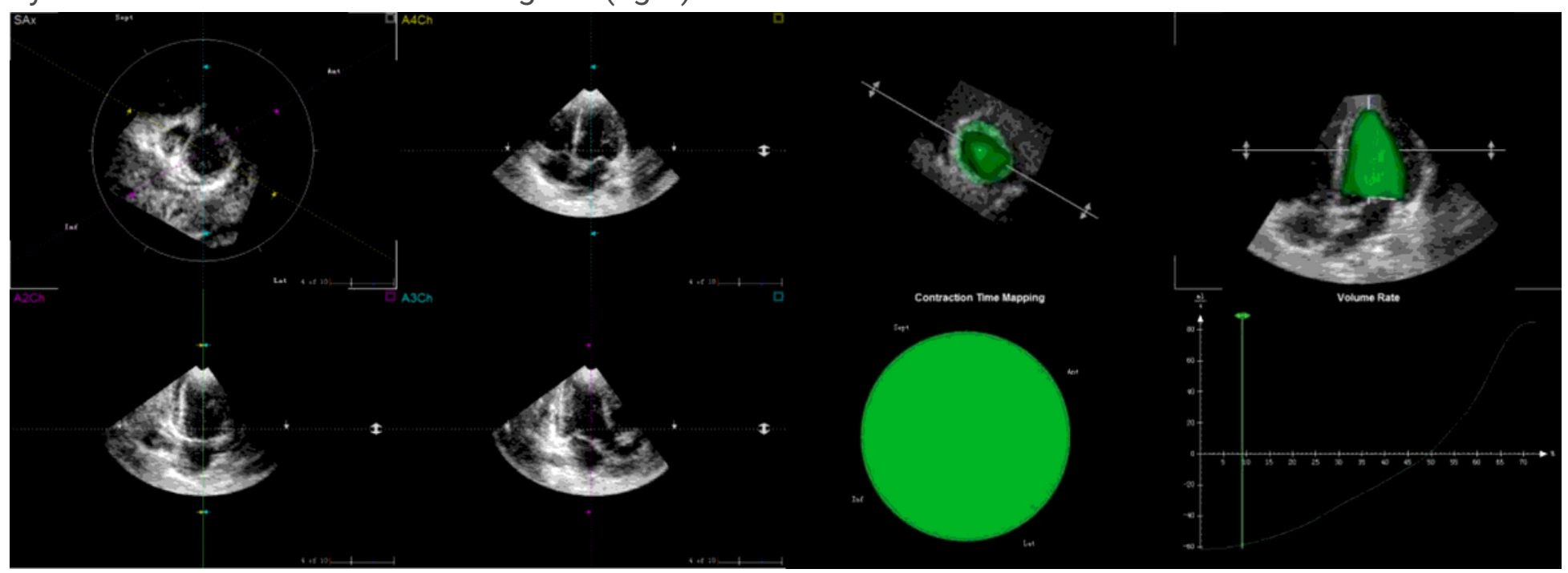

\section{Figure 2}

LV RT-3DE database analysis by TomTec LV-Function. Three standard sections of left ventricle\apical four-charmber view, two-charmber view and three-charmber view were obtained by cutting and rotating the LV three-dimensional data base (left). Left ventricular volume model was automatically generated by delineating endocardium of the three orthogonal sections, and the volume-time curve was also displayed by two-dimensional coordinate diagram (right). 


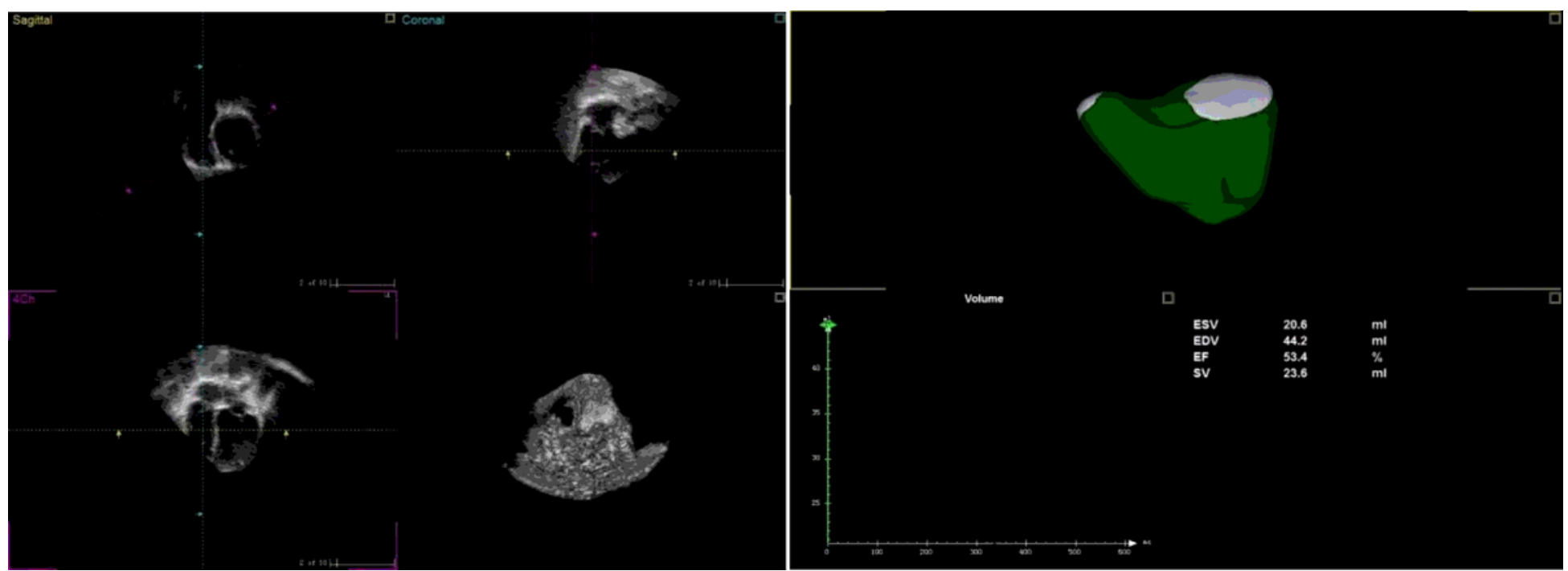

\section{Figure 3}

RV RT-3DE database analysis by TomTec RV-Function. Three orthogonal sections of right ventricular fourchamber view, short axis view, coronal view were obtained by cutting and rotating the RV threedimensional data base (left). Right ventricular volume model was automatically generated by delineating endocardium of the three orthogonal sections, and the volume-time curve was also displayed by twodimensional coordinate diagram (right).

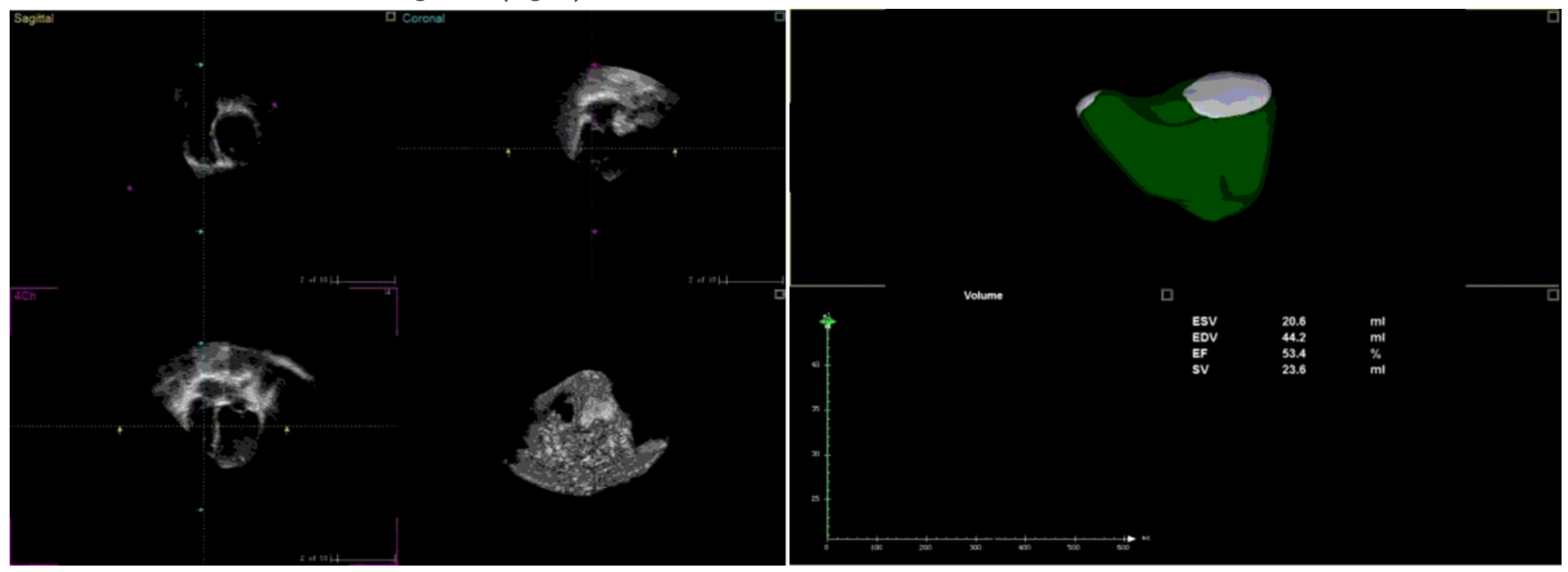

\section{Figure 3}

RV RT-3DE database analysis by TomTec RV-Function. Three orthogonal sections of right ventricular fourchamber view, short axis view, coronal view were obtained by cutting and rotating the RV threedimensional data base (left). Right ventricular volume model was automatically generated by delineating endocardium of the three orthogonal sections, and the volume-time curve was also displayed by twodimensional coordinate diagram (right). 

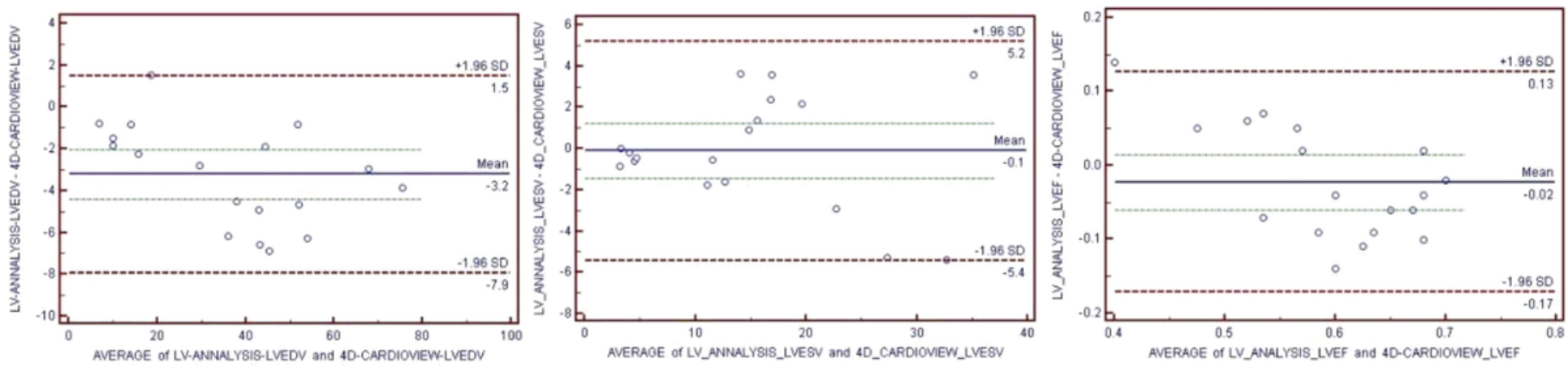

Figure 4

BA curve for TomTec LV-analysis and TomTec 4D cardio-view 2.1 eight-plane method on LVEDV, LVESV, LVEF.
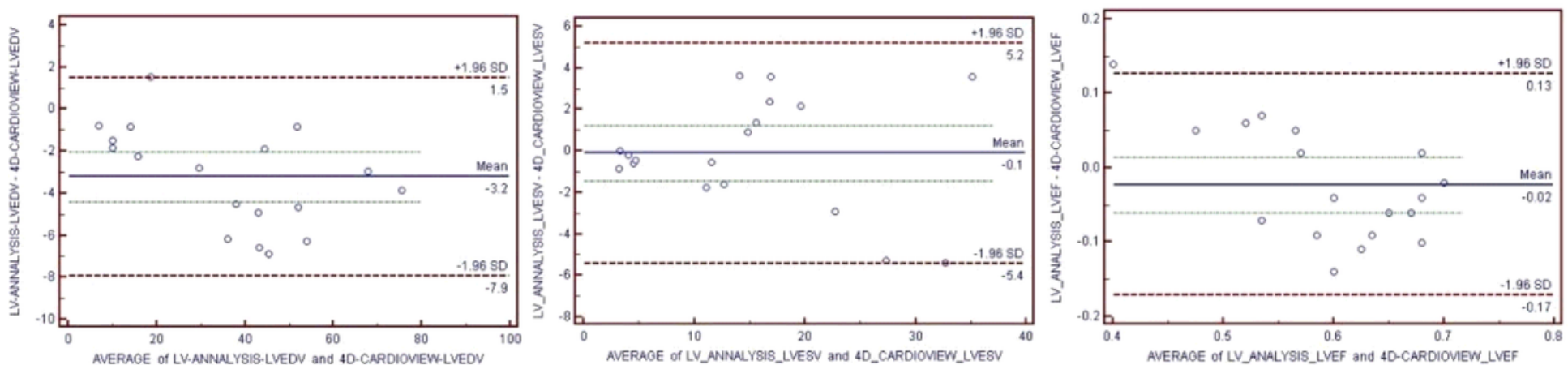

Figure 4

BA curve for TomTec LV-analysis and TomTec 4D cardio-view 2.1 eight-plane method on LVEDV, LVESV, LVEF.
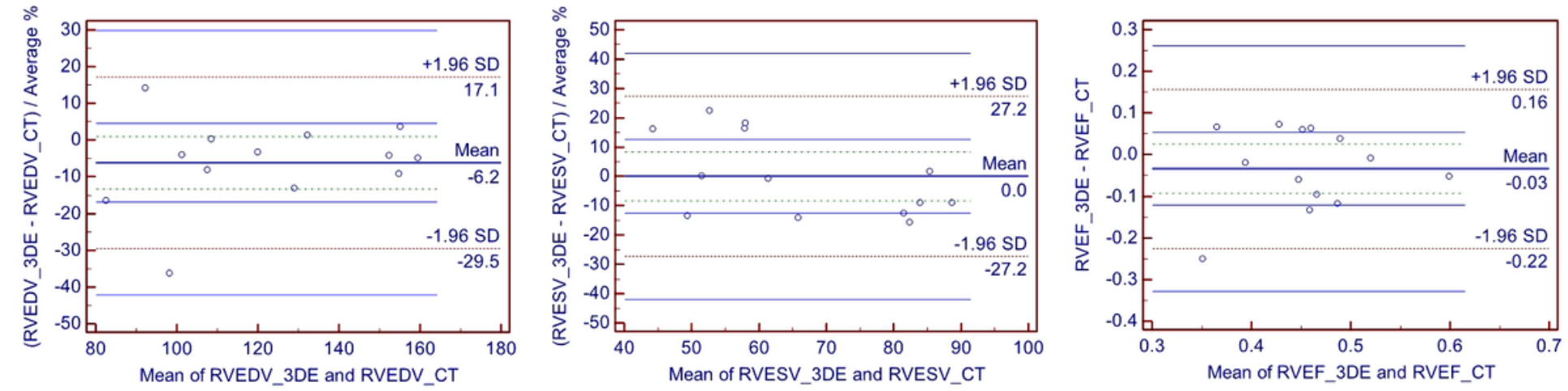

Figure 5

BA curve for TomTec RV-Function analysis and MCT on RVEDV,RVESV,RVEF 

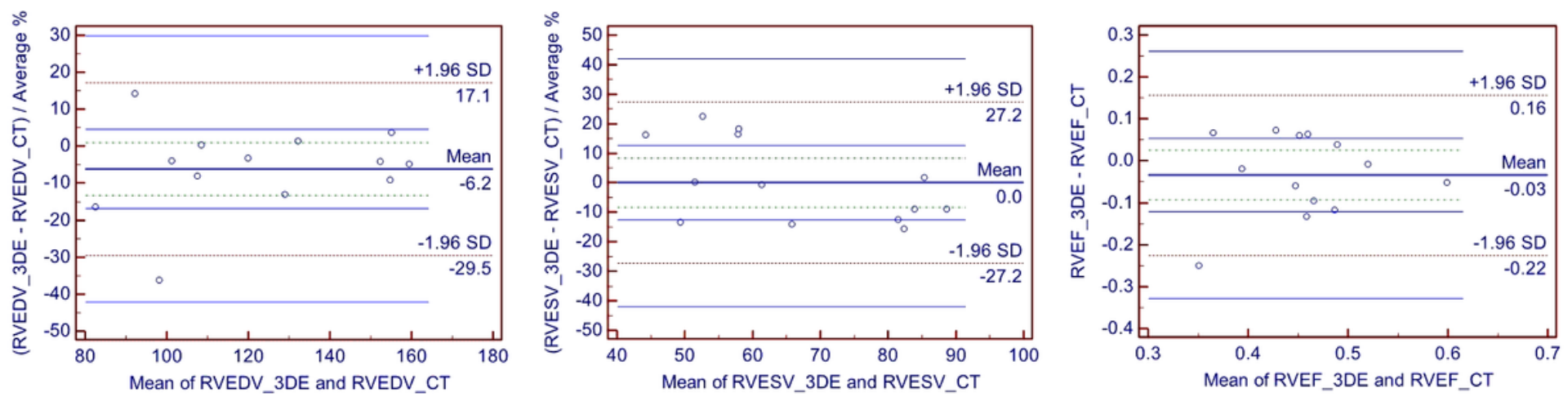

Figure 5

BA curve for TomTec RV-Function analysis and MCT on RVEDV,RVESV,RVEF

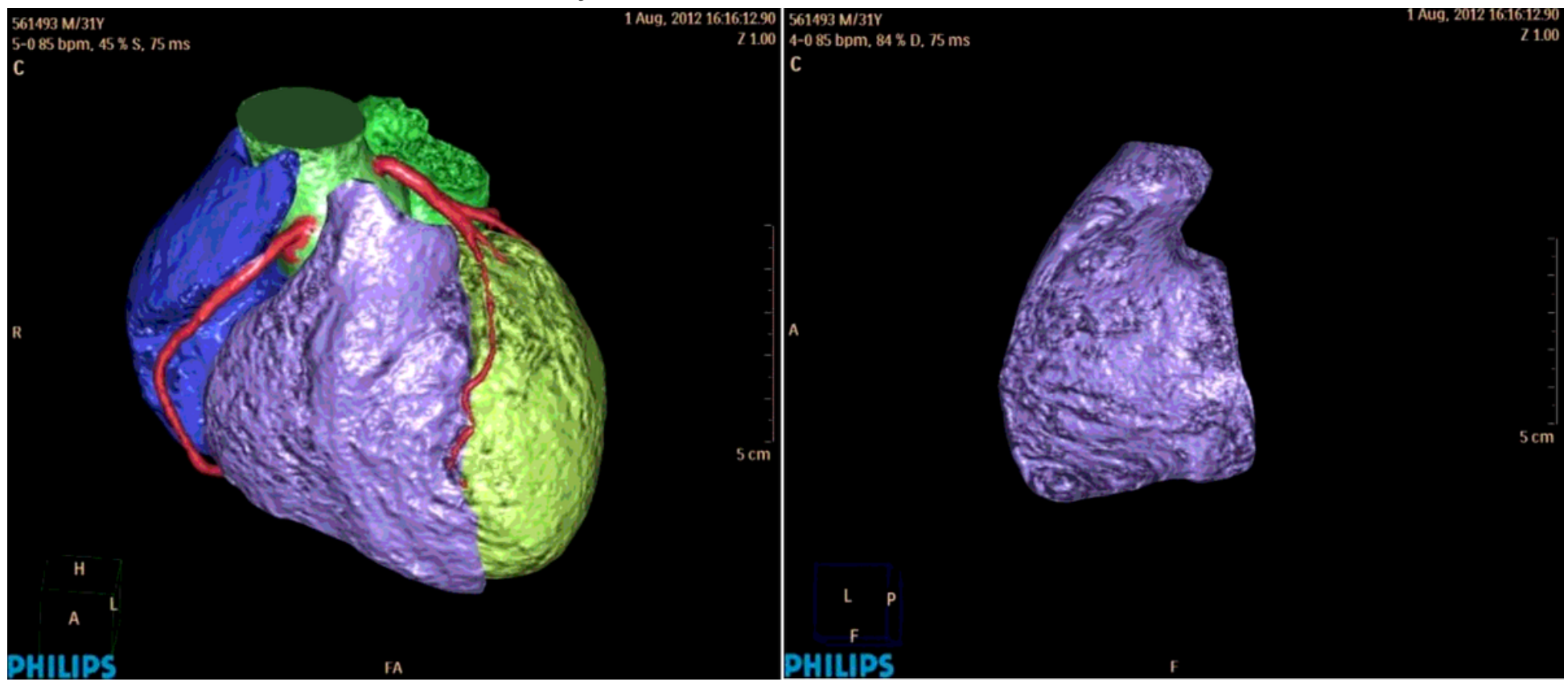

Figure 6

MCT right ventricular volume pattern (blue section) 


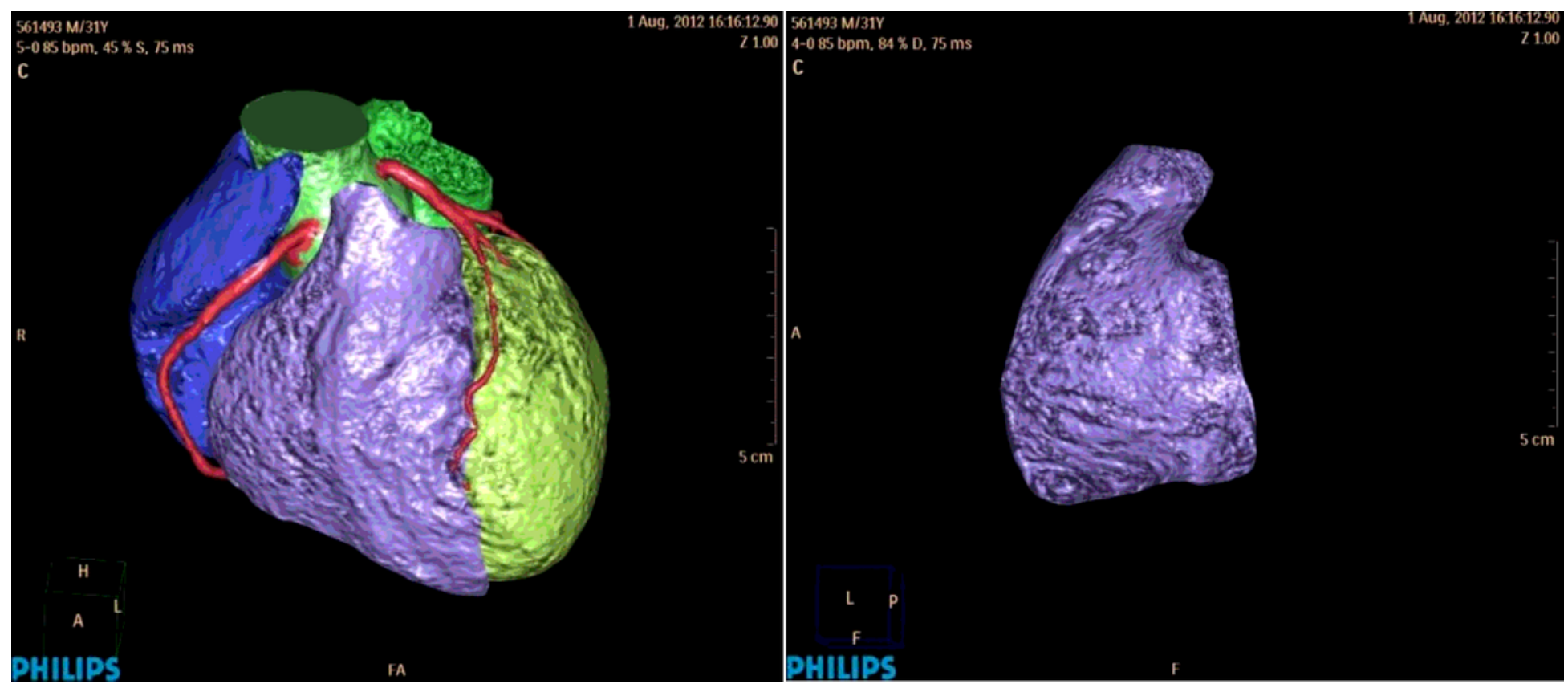

\section{Figure 6}

MCT right ventricular volume pattern (blue section) 